\title{
Adaptable and Programmable Formwork for Doubly Curved Concrete Surfaces
}

\author{
Lynn Hyun Kieffer ${ }^{1}$, Paul Nicholas ${ }^{2}$ \\ ${ }^{1}$ CITAstudio, The Royal Danish Academy of Fine Arts, Schools of Architecture, \\ Design and Conservation ${ }^{2}$ CITA, The Royal Danish Academy of Fine Arts, Schools \\ of Architecture, Design and Conservation \\ ${ }^{1}$ kieffer.lynn@outlook.de 2paul.nicholas@kadk.dk
}

This paper lays out a fabrication and simulation method for an adaptable and reusable moulding system for the production of fibre reinforced concrete elements. This research leverages soft robots and their computational controllability as means of a composite material and as such the base of a controlled and adaptable moulding system. This paper describes the development of this programmable material towards a functioning system for casting processes with fibre glass reinforced concrete. The controllable material allows to deploy target shapes and to eliminate supplementary falsework and the customized production of moulds for doubly-curved concrete elements. It also lays out a feedback method, which serves as adjustment tool of the simulation to the physical behaviour of the material as well as simulation method for target based geometries.

Keywords: adaptable moulding system, soft robotics, deployable material, programmable material

\section{INTRODUCTION}

The introduction of computational drawing methods into the field of architecture have allowed the design of complex geometries, which however often lead to complicated fabrication and construction processes. Traditionally, formwork for doubly curved concrete surfaces are produced through subtractive fabrication methods - mainly using foam or wood. (Popescu et al. 2018) These processes are material intensive, time-consuming and contribute to a large extent to the global waste production. Due to its rigidity and non-adaptability, the moulds can only be used for one single geometry and only rarely transferred or reconfigured into a different one. (Schipper 2015) In 2017, the construction industry was re- sponsible for $20-50 \%$ of the consumption of natural resources and has produced $50 \%$ of the globally accumulated solid waste. (Thomas and Costa 2017) This research challenges the contemporary construction methods for doubly curved concrete surfaces requiring custom-tailored formwork and moulds. It proposes a different approach to fabricate formwork through the deployment of a reusable, deployable and programmable composite material, as a means to reduce the solid waste associated to the construction. This composite material leverages the qualities of programmable soft actuators, which can be controlled through material grading. The actuators deploy a layer jamming mechanism - which stiffens the element when actuated through negative air- 
pressure. (Li et al. 2018) Soft robots are - in the field of production and fabrication - normally employed for safer human-machine and to reduce mechanical obsolescence, but they are also developed towards biomedical applications as well as search and rescue activities. (Saigo et al. 2018; Schmitt et al. 2018; Mahon et al. 2018) In the world of architecture these malleable robots often appear as tools, however, novel research is investigating their potential as building materials, analysing their thermal, acoustic and shape controlling qualities as well as their ability to generate interaction between users and built environments. (Decker, 2015; Kieffer and Nicholas, 2018; Farahi, 2018) Such applications remind of Smart Materials, which unlike these actuators - are actuated through external stimuli. (Addington et al. 2005) The here described materials are actuated through inflation or deflation of their own body, achieving a computationally programmed and designed deformation through tailored properties. (Bouaziz et al. 2008; Ou et al. 2018) The soft cells used in this research are unlike most soft actuators actuated through the creation of a vacuum, activating a mechanical jamming mechanism of a metamaterial. Metamaterials are artificial materials, which don't derive their behaviour from the composition of their base material but from their designed structure. (La Magna and Knippers 2018) The material described in this research leverages the reconfigurability and adaptability through modular assembly of different types of modules, categorized by curvature level in actuated state. The method of assembly of homogeneous surfaces through modules allows the creation of a large variety of actuated geometries within a rich design-space.

\section{BACKGROUND}

Although highly adapted moulds through methods such as prestressed bespoke formwork or additive manufacturing approaches allow high precision and considerable reduction of the material consumption during production process, they are mostly used as lost-formwork. This requires an additional pro- cess step during the production and doesn't allow to reuse the same construction material. Other methods to produce freeform geometries in concrete, such as additive manufacturing processes are being explored. However, most of the commonly used deposition methods of paste-like materials are bound to inclinations minor than $30-45^{\circ}$ and are time-consuming processes. (Booshan et al. 2018)

This research investigates reusable and flexible approaches for doubly-curved concrete elements, and explores such the scale of small panels. Already in the 1960's, Renzo Piano described a complex mould, which unlike rigid custom-made ones can be adapted and can thus describe the target shape. This mould is adaptable due to incorporated pistons, that move a heterogeneous surface vertically into position. (Eigenraam 2019) Similar adaptable moulding techniques have been developed by the company Adapa and at the University of Texas in Arlington. Adapa generated a flexible, controllable mould, which is able to take a minimum curvature of a radius of $400 \mathrm{~mm}$ up to infinity. A silicon membrane is fixed to a kinetic support System. The membrane can be brought into a single and double curved surface and can withstand the load of up to $16 \mathrm{~cm}$ of wet concrete. (Adapa.dk) A similar approach has been achieved by Bell et al., at the University of Texas, using a 3D-printed membrane and an actuated support. The membrane implements an origami inspired folding strategy and is printed using two different materials. These methods demonstrate non-rigid moulding examples to cast concrete or other materials on them. (Bell et al. 2014) Different methods - which enable the reconfiguration of a shape generating material and the reuse of the moulding system - can be exemplified through sub-additive processes. Here a granular material is robotically shaped to generate the negative of the target geometry, on which the paste-like material is then printed through deposition. (Zivkovic and Battaglia; 2018)While these state-of-the-art fabrication methods allow a reduced amount of material, waste and time involved in the processes; and at the same time are completely com- 
Figure 1

Assembly method of a soft-actuated module.

Figure 2

Actuation of the material and three different

geometries

characterizing the

three different

module types.
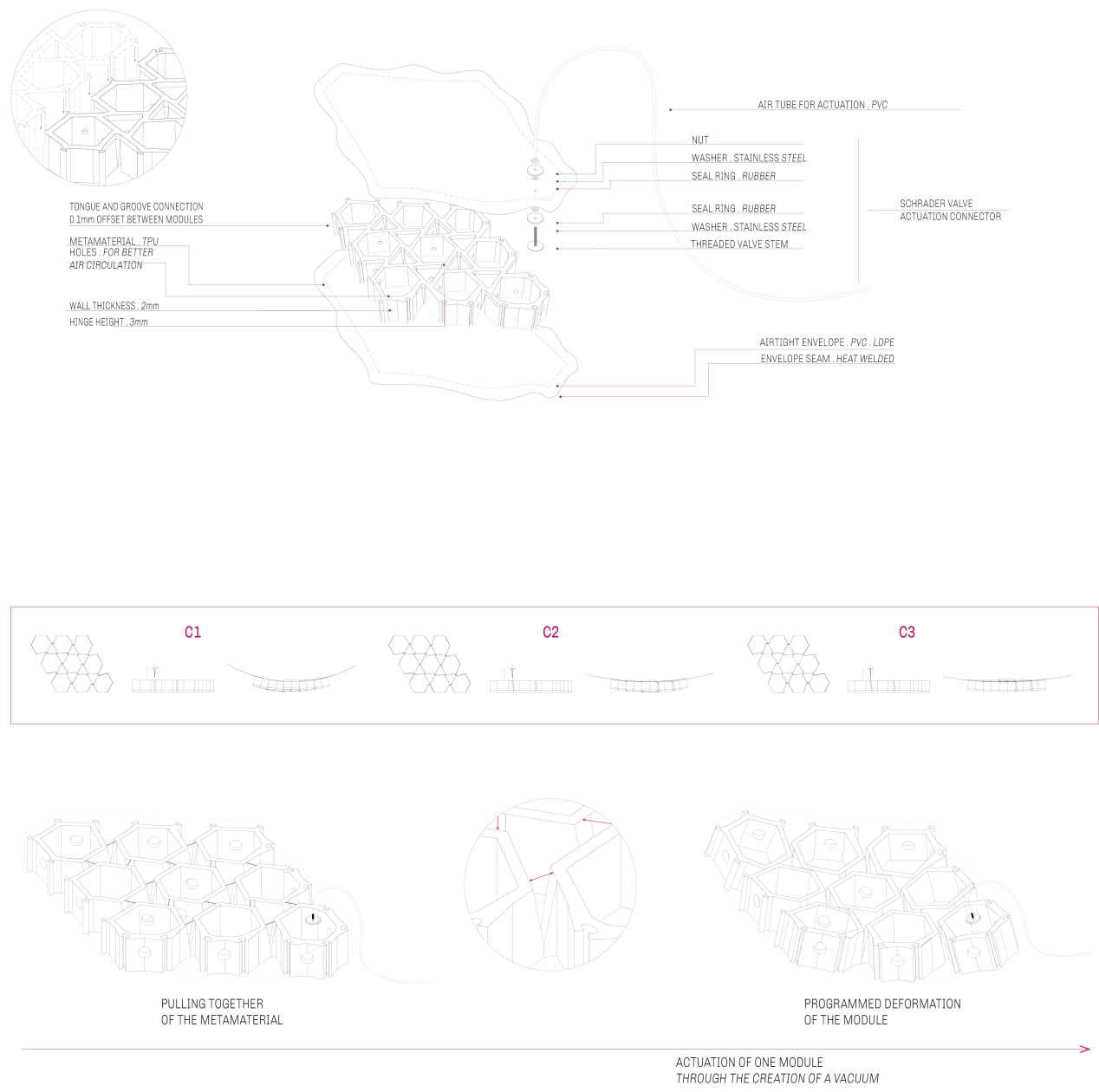
putationally controllable, they struggle with complexity and freedom of geometries.

State-of-the-art explorations of auxetic pattern, deployable systems and actuated elements investigate such programmation of geometries. Auxetic pattern can be applied as cutting pattern in plane surfaces or as generated pattern of a material to provide a planned target shape. The first approach can be exemplified amongst others by Friedrich et al., where metal sheets are gradually deformed by robot arms or through the pattern cutting and relaxation of textiles. Such processes force the material into a given spatial geometry in a controlled manner. (Friedrich et al. 2017)

A different approach, leveraging auxetic pattern for deployable structures is developed by KonakovicLukovic et al.. These auxetic assemblies are adapted to control a two-dimensional assembly towards a target geometry deploying inflation or gravity. This research demonstrates a system, where a 'spatially graded auxetic pattern' of triangular elements can be deployed towards a target shape without construction assistance or scaffolding. The bespoke system can be brought to site in planar configuration and deformed into the designed shape through expansive deployment. The fabrication process can be fully conducted in two dimensions using only 'inextensible base materials, such as fabrics, wood, metals, or plastics'. The use of deployable auxetics in architecture could allow a well-defined morpho-space. They can be fully computationally designed, and their flat, non-inflated state makes it easier to bring them to the construction site, where they are deployed through inflation. (Konakovic-Ludovic, Konakovic and Pauly, 2018)

Material grading and pneumatic actuation can also be controlled through a computationally designed, knitted envelope. Sean Ahlquist et al. have developed actuatable elements, where an encoded shape is generated through inflation of a thin silicon tube inside a graded, computationally designed knit, guiding the deformation of the element (Ahlquist, McGee, Sharmin, 2017) The described recent re- searches investigate novel construction processes, to challenge the commonly used rigid and solid formwork and moulding systems. Although all of these methods allow to reduce material use, laborintensive work and to construct at faster pace, they are bound to a single target shape. In contrary to this, the modular assembly of soft actuators proposed in this research, is able to morph between shapes within a rich morphospace.

\section{METHOD}

This paper reports the design, fabrication and simulation process for an adaptable and programmable material as a reconfigurable and thus reusable moulding system for concrete elements. This material is an assembly of dissimilar modules, placed in a grid-like system, which is actuated through a vacuum. This method allows to deploy a spatial geometry in fast pace and generate a stable formwork for concrete elements. The assembly method described is a progression of a previously published method, however, the current approach has different actuation and assembly methods. (Kieffer and Nicholas, 2018)

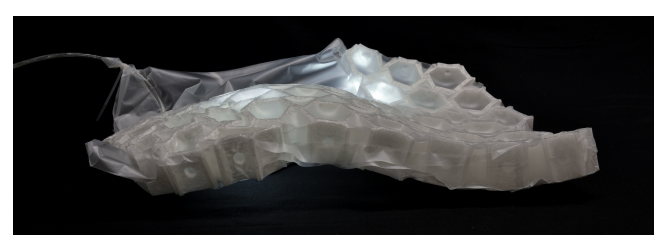

The actuatable material in this research is an assembly of multiple metamaterial modules, which are 3D printed through a Fused Deposition Modelling method (FDM) using a flexible thermoplastic Polyurethane TPU filament. The flexibility of this material allows to grade the printed geometry in a way, that certain parts of it are completely stiff and others provide hinges, that bend into the defined direction, while using one material. The printing of the metamaterial modules - which define through their geometry the deformation of the actuated elements - provides a high level of precision, which in return enables the integration of tongue and groove con-
Figure 3

Small assembly of actuatable modules. 
Figure 4

Insertion of a

Schrader Valve to

actuate the mould

Figure 5

Adjustment of the digital simulation to the physical behavior through a feedback process. nections into the modules themselves. Through this connection method, the modules can be joined and disassembled easily and form, when connected a homogeneous surface. To provide the highest stability but consume the lowest amount of material, we have found that for a printer nozzle with 0.4 diameter, an infill of $30 \%$ is needed. This needs to be raised to $100 \%$ when the larger nozzle sizes are used. In this research, the modules are printed with two perimeters to provide higher stability. For the experiments produced during this project, three different module types have been used. All of them fit within the same grid, meaning, that they are designed with the same boundary and connection conditions. Every module type is only defined by its internal geometry and its achieved curvature in deformed state. The moulding systems is adaptable through reconfiguration of the different modules and can change its curvature direction through orientation of the modules themselves.

The modular assembly is then wrapped by an airtight envelope. This is an elemental part of the material system, as it creates the actuatable environment. For this, different material performances have been tested. The desired material needs to be airtight, creating a hull - either through gluing or heat welding of the sheet material. Furthermore, it is required to be elastic enough to completely fulfil the actuation, but stiff enough to prevent the material from ripping if the internal negative pressure is too low or being punctured by sharp objects during the production process of the concrete elements. Furthermore, the elasticity needs to be very limited, to prevent the material from expanding, while the concrete is sprayed on it. If this would be the case, the mould can't show the desired stability and the casting fails. Considering these requirements, we chose to use a $0.5 \mathrm{~mm}$ thick PVC sheet, which is a thermoplastic material and can therefore be welded using heat.

A Schrader ventil has been introduced into the envelope to provide the best solution without weakening the airtight hull. At the same time this method is able to prevent air to stream back into the assembly, which would cause it to lose shape.
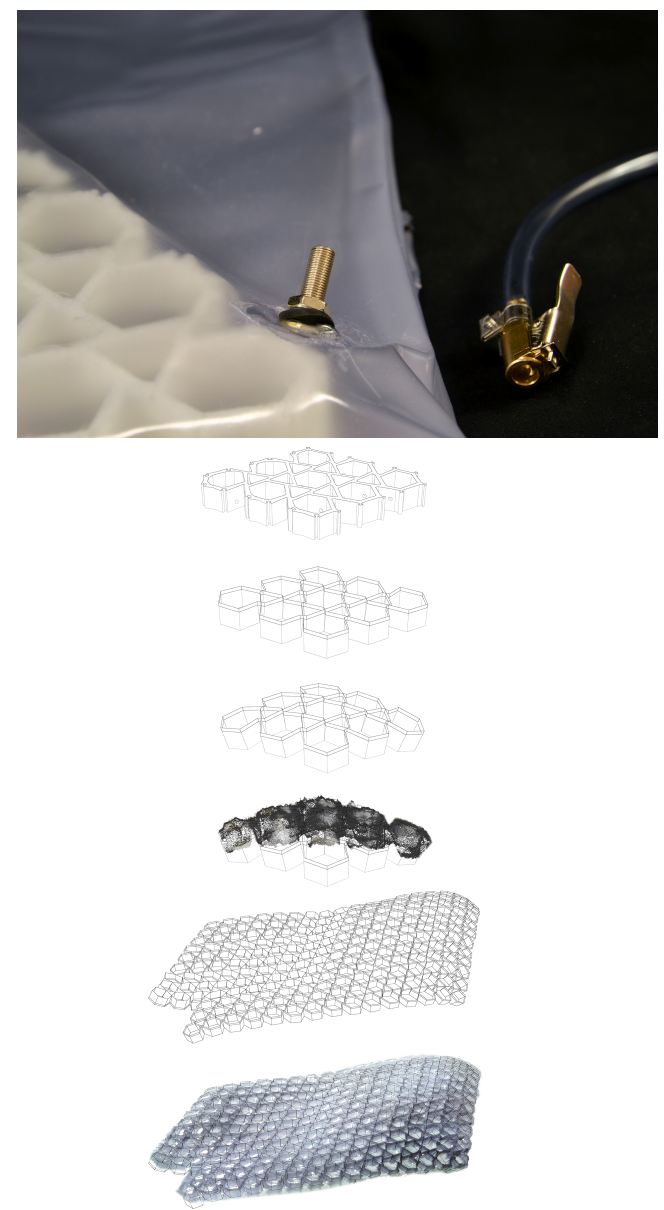

The approximation of geometries can be predicted through a spring-based simulation. For this method the parameters of placement and orientation of modules in the grid as well as their module and curvature type are being fed into the simulation definition. This process has been previously described. (Kieffer and Nicholas, 2018) This method, however, is an approx- 
imation of the physical deformation of an assembly - but if implemented into an evolutionary solver can provide us with an idea of the possible design space of an assembly of a set of modules. A novel addition to this simulation method is the adjustment of the computational simulation to the physical behaviour of the actuated assembly through a feedback loop. This has been done through the scanning of individual, actuated modules and the simulation of the geometries that have been designed to print. This simulation has been developed as a spring-based simulation of lines, to increase the calculation speed. The physical actuated module has been scanned using a Faro laser scanner. This high precision engine generates a spatial point-cloud, which can then be digitally read and used to compare to the simulated model. The simulation of the individual modules has then been adjusted to the scans through forces of springs. After the right adjustments for individual modules has been accomplished, the same process has been made for larger assemblies. The prediction of actuated geometries can now be predicted to a precision of $+-25 \mathrm{~mm}$ - if the parameters of placement, orientation and module type are given. This method allows a high precision formfinding process, through which geometries can be searched for a given set of metamaterial modules.

A different approach to simulate geometries has been developed to identify the necessary parameters for a target geometry. For this simulation process, a designed surface is fed into the simulation definition. The input is planarized and mapped onto the grid of modules. The modules boundaries which lie within the planar surface are mapped back onto the spatial one. This spatially deformed grid then serves as analysis base for the curvature and orientation of the modules. In a next step this information is then converted into a discretized pattern of modules - chosen from a library of module types, sorted according to their curvature.

First experiments with this material have been made in small scale and with gypsum as casting material. In first probes, the gypsum mass had not been reinforced, which leads to rupture of the material during the drying process, as this one shrinks slightly during the curing process. However, the mould doesn't allow this slight movement, as the stiffening of the vacuum can't be ceased during the curing time, to prevent deviations from the given geometry of the mould. In order to eliminate this problematic of rupture of the casted material, a simple jute textile sheet has been inserted onto the mass.
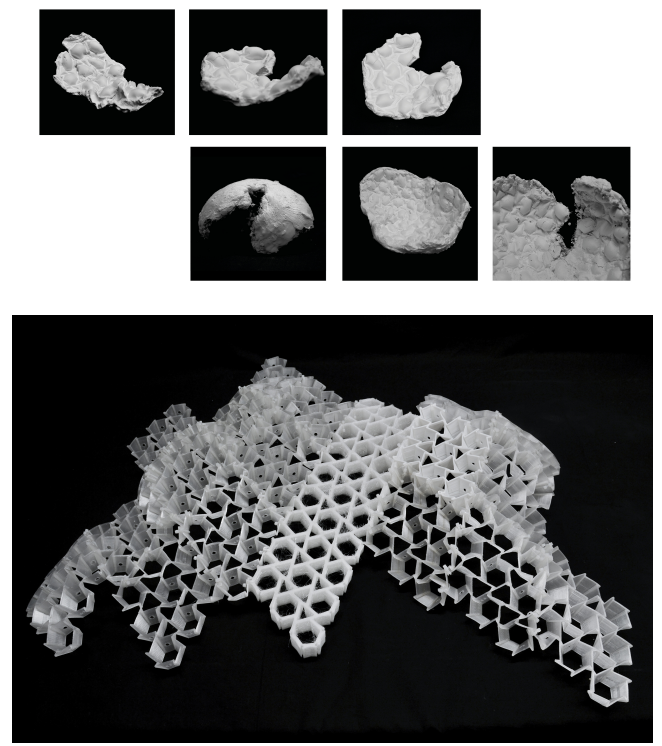

Figure 6

Target based simulation method.

Figure 7

First probes using gypsum as casting material on the adaptable mould.

Figure 8

Assembly of metamaterial modules, to create the geometry generating skeleton of the mould. 
Figure 9

Fabrication process

of concrete

prototypes in

collaboration with

BBFiberbeton to

evaluate and

analyze the

adaptable mould.
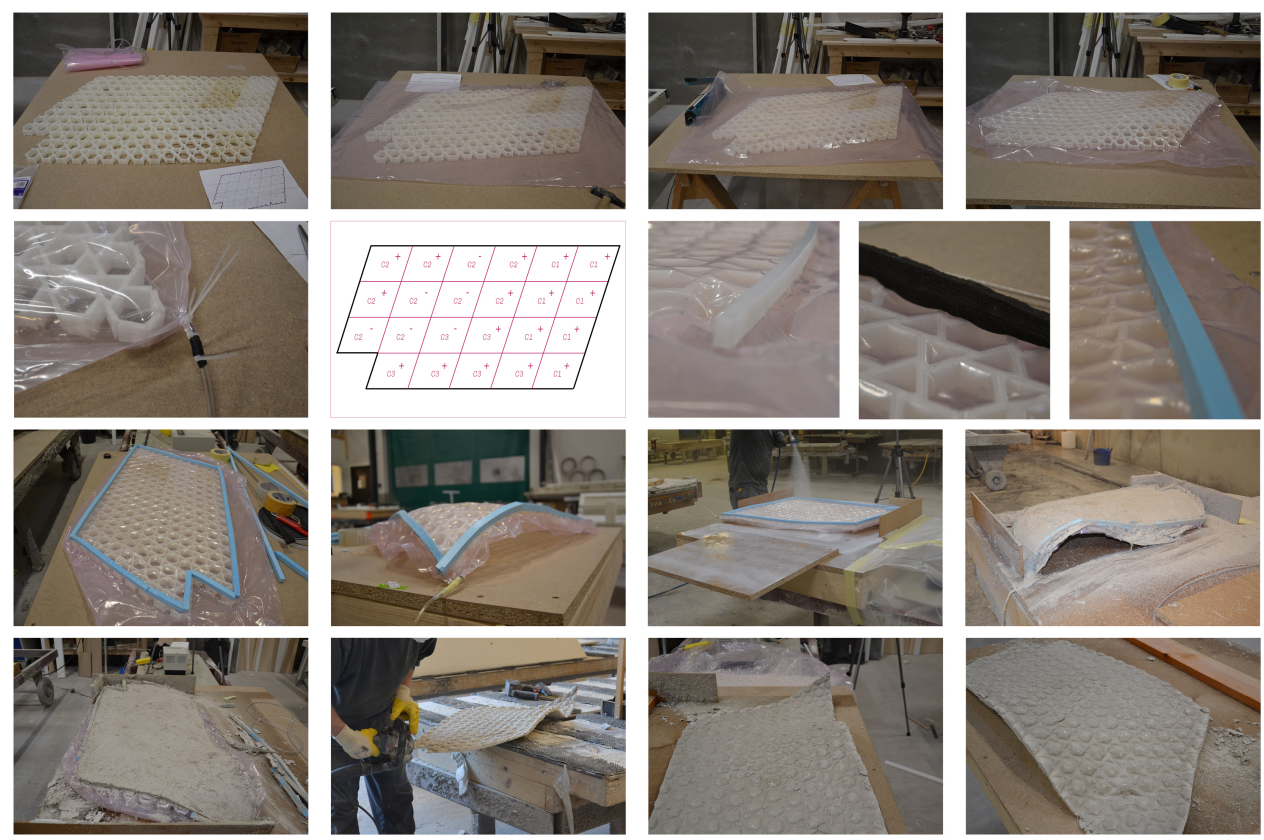

The thickness of the metamaterial modules has been increased from $1.5 \mathrm{~mm}$ to $2.5 \mathrm{~mm}$ - for modules of $150 \times 250 \mathrm{~mm}$, to prevent the assembly to be deformed in a undesired manner through the application of the material load during the casting process, but also through continuous vacuuming of the mould.

In a successive step, one of these moulds has been tested in collaboration with BBFiberbeton, a Danish company specialized on adaptable moulds and glass fibre reinforced concrete (GFRC). The envelope of the first mould tested still used an LDPE foil of 200 micron thickness. Although this material provided good results in use for the gypsum models, many undesired details are imprinted into the concrete, as the thin foil crumples slightly when actuated instead of performing as a smooth surface. A different issue was the deflection of the mould during the spraying and curing of the concrete. The negative pressure inside of the assembly was not low enough to keep a high stability and the load of the sprayed material caused it to slowly sink in slightly. This was also due to tiny punctures in the envelope, that caused minor leaks and prevented full stability as well as small leaks in the heat welded seam. In a second iteration, the envelope material has been exchanged into the previously described PVC sheet. The $0.5 \mathrm{~mm}$ thick material prevented from being punctured at any point and the heat welded seams seemed to be much stronger than its predecessors. The casting on theses super smooth surfaces doesn't require release agent, which might accumulate in the valleys of the 
mould when actuated and cause undesired irregularities on the concrete surface. The general problem, that we ran into in both iterations was the problem of constant actuation of the mould during the full curing process. The lack of this and slight movements of the mould as well as internal geometrical differentiations over time caused small cracks, delamination of layers or complete breaking of smaller pieces on the concrete surface. The manually produced hulls always have certain tolerances and bear tiny irregularitites in the welded seams, therefore to stabilize the target geometry - at this stage of the research the mould needs to be attached to a constant source of air suction.

As this could not be provided due through a small vacuum pump, the third and final iteration has been executed using a Venturi pump. This mechanism allows to use the internal compressing system of the factory and to convert it into a negative air pressure. For this last iteration of prototyping we deployed this system and constantly actuated the mould with the internal compression system running at approximately 8-9 bar for 15 hours. This method allowed the mould to be constantly stable without the possibility of slight deformation and related delamination or displacement of fibres in the mix. After curing of the approximately $20 \mathrm{~mm}$ thick concrete mass, the air pressure in the mould can be raised - which softens the assembly material - and allows the demoulding of the casted element. The PVC plastic can then be cleaned using only a sponge, clean water and soap, which allows the reuse of the exact same set of components of the mould for further production processes. The geometry of the target element can then be reconfigured through following the assembly parameters given by the simulation. Through this method, the mould can be reconfigured and adapted in a controlled manner and reused for multiple casting processes.
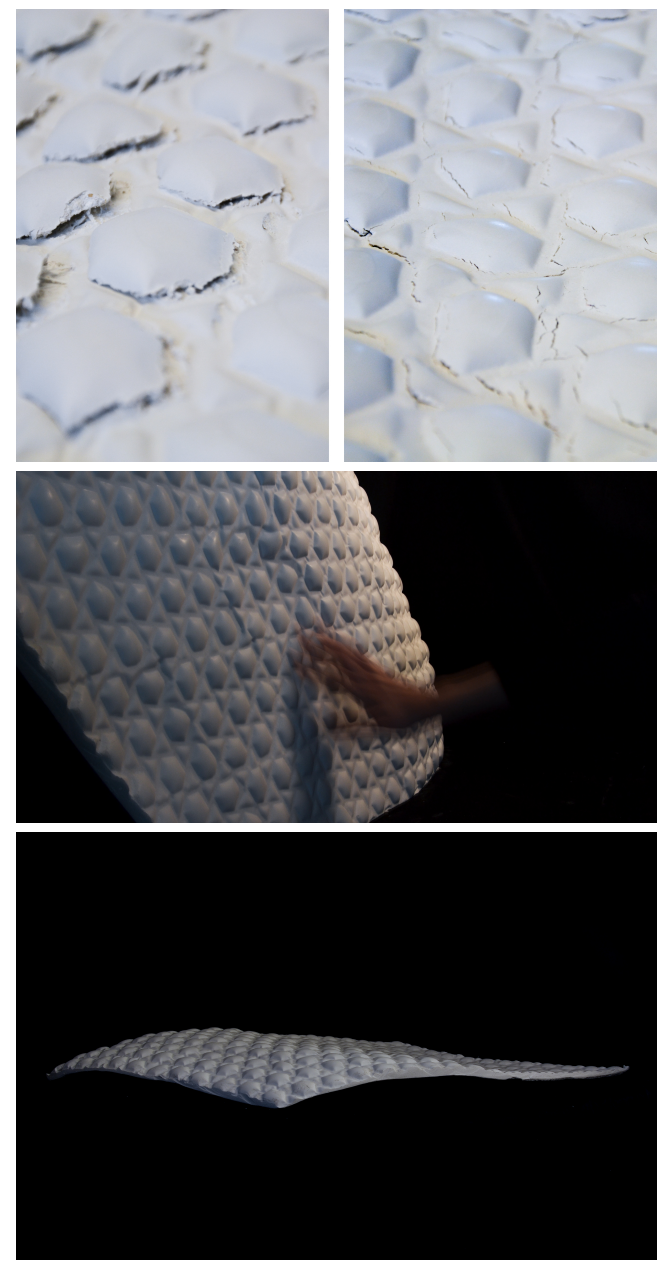

Figure 12

Concrete prototype from third iteration using the Venturi method. 
Figure 13

Concrete prototype from third iteration using the Venturi method.

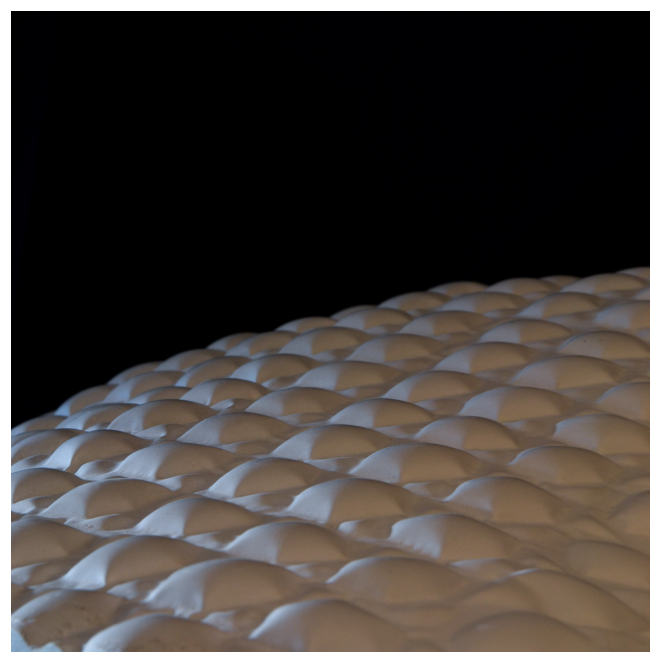

\section{Conclusion and Reflection}

The adaptable and computationally controllable material system described in this research and deployed as a moulding system has been developed towards a functioning system for approximately $20 \mathrm{~mm}$ thick panels. The programmability and the adjustment of the simulation to the physical behaviour of the deformed assemblies allows for high precision in the production process. The same set of items can be reused to generate large variety of different geometries and can therefore considerably reduce the fabrication time for complex moulds and at the same time decrease the material consumption to a minimum. In case that the metamaterial modules wear out or are damaged, they can even be recycled and used to create new 3dprinting material. At the same time, the use of a material system which is actuated through a vacuum reduces the complexity of mechanical hinges and pistons of mechanically adaptable moulds. The modular assembly of this system also enables easy joining and scaling of the moulds and therefore is not necessarily bound to a predefined limit. The fabrication method for this moulding system enables quick deployment of a flat, programmed surface, but only requires simple tools. In prospective to the state of this research, it would be interesting to scale up the production and increase the size of the moulds and eventually think about it as monolithic formwork for full scale architecture.

\section{ACKNOWLEDGMENTS}

This paper presents a part of Lynn Kieffer's research project, developed at CITAstudio at the Royal Danish Academy of Fine Arts, School of Architecture, and supervised by Prof. Paul Nicholas. The authors would like to thank the team of BBFiberbeton for the great collaboration, which enabled the testing of this fabrication method.

\section{REFERENCES}

Addington, M. and Schodek, D. 2005, Smart materials and technologies, Elsevier / Architectural Press

Booshan, S., Ladinig, J., van Mele, T. and Block, P. 2018 'Function Represetation for Robotic 3D Printed Concrete', : ROBARCH 2018, Robotic Fabrication in Architecture, Art and Design 2018

Bouaziz, O., Bréchet, Y. and Embury, J. 2008, 'Heterogeneous and Architectured Materials: A Possible Strategy for Design of Structural Materials', Advanced Engineering Material, 10, pp. 24-36

Brad, B., Barnes, N., Ede, A. and Cord, T. 2014 'Casting non-repetitive geometries with digitally reconfigurable surfaces', Design Agency [Proceedings of the 34th Annual Conference of the Association for Computer Aided Design in Architecture, Los Angeles, pp. 453-462

Decker, M. 2015 'Soft Robotics And Emergent Materials In Architecture', In Real Time: Proceedings of the 33rd International Conference on Education and Research in Computer Aided Architectural Design in Europe

Eigenraam, P. 2019, Flexible muld for production of double-curved concrete elements, Master's Thesis, TU Delft

Farahi, B. 2018 'Heart of the Matter: Affective Computing in Fashion and Architecture', Recalibration: On imprecision and infidelity Paper proceedings book for the 2018 Association of Computer Aided Design in Architecture Conference

Friedrich, J., Pfeiffer, S. and Gegnagel, C. 2017 '). Locally Varied Auxetic Structures for Doubly-Curved Shapes', Humanizing Digital Reality Design Modelling Symposium Paris 2017 
Kieffer, L. and Nicholas, P. 2018 'Pneumatically Actuated Material | Exploration of the Morphospace of an Adaptable System of Soft Actuators', Recalibration: On imprecision and infidelity Paper proceedings book for the 2018 Association of Computer Aided Design in Architecture Conference

Konakovic-Lukovic, M., Konakovic, P. and Pauly, M. 2018 'Compuational Design of deploayable auxetic shells', Advances in Architectural Geometry 2018

Li, S., Vogt, D. M. and Wood, R. J. 2018 'Fluid-driven origami-in $\neg$ spired artificial muscles', Proceedings of the National Academy of Sciences of the United States

La Magna, R. and Knippers, J. 2017 'Tailoring the Bending Behaviour of Material Patterns for the Inducation of Double Curvature', Design Modelling Sympoisum Paris 2017: Humanizing Digital Reality

Mahon, S., Roberts, J, Sayed, M., Chun, D., Aracri, S., McKenzie, R., Nemitz, M. and Stokes, A. 2018 'Capability by Stacking: The Current Design Heuristic for Soft Robots. Biomimetics', no source given

Ou, J., Ma, Z., Peters, J., Dai, S., Vlavianos, N. and Ishii, H. 2018 'KinetiX - designing auxetic-inspired deformable material structures', Computers \& Graphics

Popescu, M., Reiter, L., Liew, A., van Mele, T., Flatt, R.J. and Block, P. 2018 'Building in concrete with a knitted stay-in-place formwork: Prototype of a concrete shell bridge,Structures', no source given

Saigo, H., Naruse, M., Okamura, K., Hori, H. and Ojima I. 2018 'Category theory as a foundation for soft robotics', no source given

Schmitt, F., Piccin, O., Barbé, L. and Bayle, B. 2018, 'Soft Robots Manufacturing: A Review', Frontiers in Robotics and Al, 5

Thomas, N. and Costa, D. 2017 'Adoption of environmental practices on construction sites. Ambient Construído', no source given

Zivkovic, S. and Battaglia, C. 2018 'Rough Pass Extrusion Tooling', Recalibration: On imprecision and infidelity Paper proceedings book for the 2018 Association of Computer Aided Design in Architecture Conference, Mexico City

[1] http://block.arch.ethz.ch/brg/project/knit-candelamuac-mexico-city?fbclid=IwAROVD7IHzYnN2wpAgca5 XNdh41PkFe_bXdgtEvlgFOK_3CQ7Kww-m3JkY6o

[2] http://adapa.dk/wp-content/uploads/2017/01/Ada ptive-Mould-Compo囚 $\neg$ nents-A4.pdf 